\title{
Revisión \\ Enfoque de la
detención de \\ Julian Assange prensa digital de tres continentes
}

Jessica Trujillo Mero igtrujillo.fpcs逐unach.edu.ec Julio Bravo Mancero jbravo@unach.edu.ec

\section{Carlos Larrea Naranjo carloslarrea@inach.edu.ec}

Recibido: 27 de abril de 2021 | Aceptado: 20 de julio de 2021

DOI: https://doi.org/0.18272/pd.v5i1.2256

Referencia de este artículo:

Trujillo, J., Bravo, J. y Larrea, C. (2021). Enfoque de la detención de Julian Assange abordada por la prensa digital de tres continentes. \#PerDebate, volumen 5 (pp. 48-69). Quito: USFQ Press.

Jessica Trujillo Mero, licenciada en Ciencias de la Comunicación Social, Universidad Nacional de Chimborazo; mejor egresada de su promoción. Ha recibido reconocimientos institucionales por su rendimiento académico en grado, durante los años de estudio. Ganadora de varios concursos relacionados con la comunicación y periodismo.
Julio Bravo Mancero, comunicador con doctorado en Comunicación e Información Contemporánea (cum laude), Universidad Santiago de Compostela, España. Experto en metodologías cualitativas y participativas. Director de Carrera de Comunicación de la Universidad Nacional de Chimborazo (Unach).
Carlos Larrea Naranjo, doctorando en Comunicación por la Universidad de La Habana, Cuba. Autor del proyecto de creación de la Carrera de Comunicación Social, coordinador de investigación de las carrera de Comunicación. Profesor titular de la Universidad Nacional de Chimborazo (Unach). Docente de grado y posgrado. Expositor en eventos nacionales e internacionales.
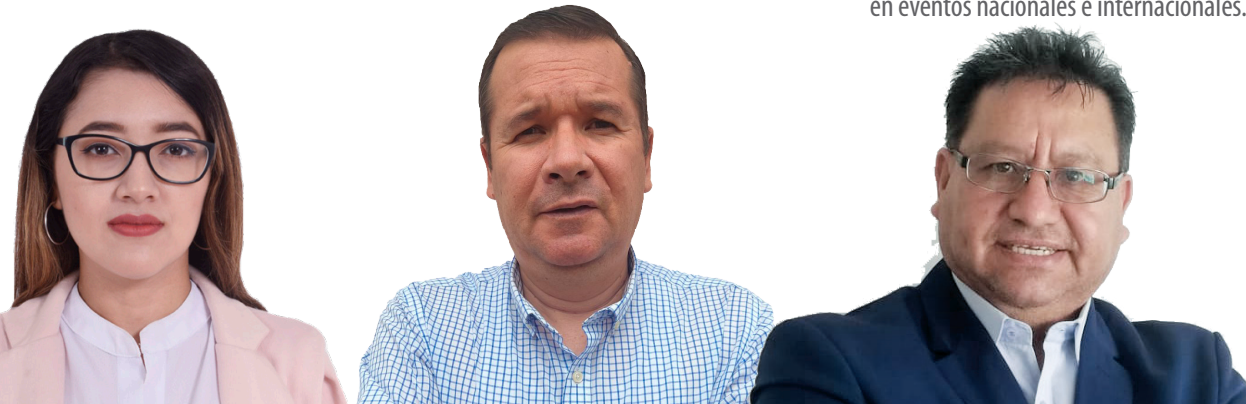


\title{
Resumen
}

Este artículo investigó el tratamiento informativo que tuvo la detención de Julian Assange luego de que el presidente de Ecuador, Lenin Moreno, le retirara el asilo político. Analizó las ediciones digitales de los diarios Komsomolskaya Pravda, El País, The New York Times y The Sun. Empleó una metodología mixta (cualitativa y cuantitativa); mediante la revisión documental recogió la información relacionada, determinando como muestra 88 contenidos. La sustentación teórica está en medios de comunicación y autores expertos. Los resultados evidencian un medio a favor de Assange, uno en contra y dos que se acercan a la imparcialidad.

\section{Palabras clave}

ediciones digitales, periodismo, tratamiento informativo, Julian Assange, prensa

\section{Approach to the arrest of Julian Assange addressed by the digital press of three continents}

\author{
Abstract \\ This article investigates the informative treatment of the detention of Julian Assange \\ after the president of Ecuador withdrew his political asylum, the digital editions of \\ the newspapers Komsomolskaya Pravda were analyzed, The Country, The New York \\ Times and The Sun. The methodology used was of a mixed type: qualitative and \\ quantitative, through the documentary review the related information was collec- \\ ted, determining 88 contents as a sample. The theoretical support is in media and \\ expert authors. The results show: one in favor of Assange, one against and two see- \\ king to approach impartiality.
}

\section{Keywords}

digital editions, journalism, informative treatment, Julian Assange, press. 


\section{Introducción}

Este artículo aborda la forma en que los medios trataron la información referente a la detención de Julian Assange. Su caso no es reciente, ha sido tema de discusión por tener conflictos con países como Suecia, donde se lo acusa de abuso sexual y solicitan su extradición, razón que lo llevó a pedir asilo en la embajada de Ecuador en Londres, y Estados Unidos, país que se sumó al pedido de extradición tras haberse filtrado material sobre la guerra de Afganistán e Irak en WikiLeaks, sitio que Assange fundó. El tratamiento informativo puede variar dependiendo de los intereses que tiene el medio; eso se evidencia en las fuentes que emplea, elementos de ayuda gráfica y audiovisual, géneros periodísticos, emplazamiento, su posición. Los diarios analizados presentan columnas, comentarios y un editorial donde reflejan su postura ante el hecho.

La pregunta de investigación planteó ¿cómo se realiza el tratamiento de la información sobre la detención de Julian Assange en las ediciones digitales Komsomolskaya Pravda (Rusia), El País (España), The New York Times (Nueva York) y The Sun (Reino Unido)? El objetivo consistió en determinar ese tratamiento mediante la identificación de los géneros periodísticos empleados por los medios al abordar el caso de Assange. Para eso se utilizó una metodología mixta (cualitativa y cuantitativa) con técnicas como la revisión documental y el análisis de contenido.

Actualmente los diarios poseen ediciones digitales en las que se accede directamente a la información. Esta característica permite encontrar contenidos publicados hace meses, algo importante para esta investigación, puesto que a Julian Assange se lo detuvo el 11 de abril de 2019 y se necesitó de una revisión a sus ediciones. Según Edo (2009, p. 36), a partir de 1995 los medios impresos fueron incorporándose a la red; inicialmente tuvieron que trasladarse del papel a la web y añadir a sus contenidos elementos sonoros y visuales que antes eran exclusivos de la radio y televisión. Con la tecnología actual, esto resulta sencillo hacerlo para complementar la información.

Es el primer caso en la historia en el que se registra un asilo político de casi siete años (2.487 días). Ecuador ha estado en consideración del mundo por haber sido el precursor del hospedaje de Assange: empezó con el gobierno del expresidente Rafael Correa, continuó luego de la llegada del exmandatario Lenín Moreno, quien en 2019 puso fin a sus beneficios. Hay opiniones variadas sobre la causa que llevó a Moreno a tomar esa decisión; los medios analizados tienen una explicación a la interrogante desde sus perspectivas y fuentes consultadas.

Los resultados de la investigación demuestran una inclinación de los medios. Solo dos de ellos se acercaron a la imparcialidad, pero los dos restantes no logran dar un equilibrio a sus contenidos: uno está a favor y el otro en contra. El 
género que predominó fue la crónica, presentando como principal característica hipervínculos en sus publicaciones.

La fundamentación teórica se evidencia en los preceptos de Astudillo y Buitrón (2005), BBC News Mundo (2019); González (2009); Ramírez (2018); Briggs (2007); Edo (2009); Di Palma (2010); Pérez (2012); RT (12 de abril de 2019) y Talavera (2012).

\section{The Alew lyork Times}
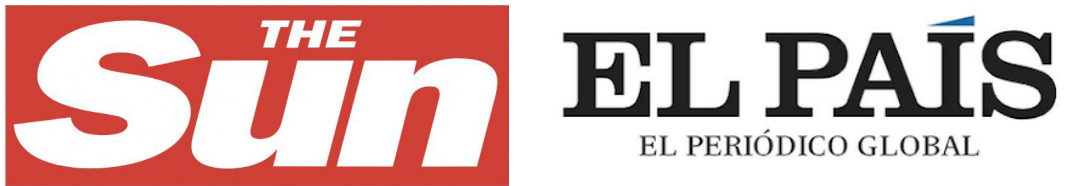

EL PERIÓDICO GLOBAL

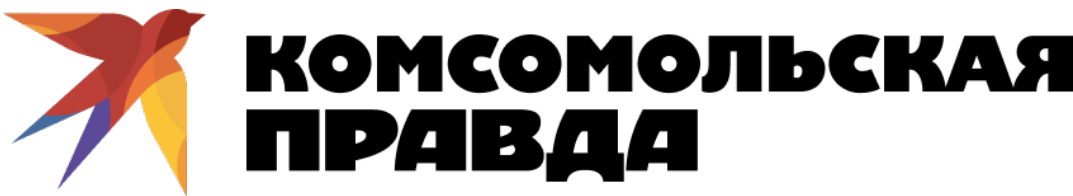

Imagen 1. Los diarios digitales analizados para esta investigación fueron Komsomolskaya Pravda (Rusia), El País (España), The New York Times (Estados Unidos) y The Sun (Reino Unido).

\section{Marco teórico. Assange: contexto histórico}

La información acerca de la vida personal de Julian Assange es muy escasa. Se conoce que nació el 3 de julio de 1971 en Australia, es programador y periodista. Es fundador del sitio WikiLeaks en 2006; allí se publican documentos e información de carácter reservada, revela evidencias clasificadas como fue la filtración de material del Ejército de Estados Unidos sobre las guerras de Afganistán e Irak de 2010, el espionaje a los tres últimos presidentes de Francia por parte de la Agencia Nacional de Seguridad de Estados Unidos, y demás casos que han hecho que WikiLeaks sea un nombre conocido.

El 19 de junio de 2012 se concedió a Assange refugio en la embajada ecuatoriana en Londres, dado que el Tribunal Supremo británico autorizó su extradición a Suecia, donde se lo acusa de abuso sexual, cargo que él negó.

La nacionalidad ecuatoriana que se le había concedido a Assange fue confirmada en 2018 por el expresidente Lenín Moreno, quien comentó que el 12 de 
diciembre de 2017 la excanciller María Fernanda Espinosa había expedido la nacionalidad para proteger a Assange y evitar su extradición a Suecia por el cargo de abuso sexual.

Las condiciones en que vivía Julian Assange en la embajada las abordó RT (2019), un canal de noticas ruso, que describe la habitación con medidas de 4 metros por 4,6 metros, donde contaba con algunos beneficios para comunicarse como computadora, teléfono e internet, también beneficios personales, cocina, ducha, lámpara, cinta de correr. Muchas de esas ventajas se redujeron con la llegada del expresidente de Ecuador, Lenín Moreno, que empezó a restringir el uso de internet y el permiso a sus visitantes.

\section{Detención de Julian Assange}

El 11 de abril de 2019 la noticia de la detención de Julian Assange apareció en los medios, luego de que el expresidente ecuatoriano Lenín Moreno declarara la retirada del asilo político y diera paso a las autoridades británicas a disponer de Assange. Según BBC News Mundo (2019), Moreno había expresado en un video que Assange presentaba una conducta "irrespetuosa y agresiva", además de haberse involucrado en temas de otros Estados, y Ecuador no quería tener problemas con países en los que intervenía. Moreno dijo eso como justificación a su decisión. Si el activista es enviado a EE. UU. podría sufrir torturas o hasta la pena de muerte.

\section{Tratamiento informativo}

La manera en que se maneja una información, considerando el enfoque, las fuentes, los datos y la inclinación que tendrá, depende de los intereses y políticas del medio; lo ideal es lograr un equilibrio en las notas y acercarse lo más posible a la imparcialidad. Según Talavera $(2012$, p. 3), este tratamiento informativo en los medios masivos se torna complejo porque también influye la especialización de los periodistas.

\section{Periodismo}

La labor realizada en esta profesión debe ir encaminada según lo que la sociedad necesita saber, haciendo un levantamiento de información de los hechos más relevantes y entregando un contenido lo más cercano a la realidad posible. Astudillo y Buitrón (2005, p. 17) hablan de la vocación que debe sentir el periodista, vista como un sacerdocio, un llamado, entregarse a la búsqueda de la verdad y su difusión sin importar los riesgos que existan.

\section{Periodismo digital}

La tecnología forma parte de la vida de los seres humanos. Los diarios no solo informan mediante impresos, ahora difunden los resultados de su trabajo en la web, en sus presentaciones en la red añaden elementos que 
enriquecen el texto y pueden cobrar vida con la tecnología. El periodismo digital se complementa con videos, fotos, audios, infografías, capturas e hipervínculos. Los diarios estudiados presentan todos esos elementos de ayuda gráfica y audiovisual. "Los lectores ya no son los receptores pasivos de nuestros mensajes. Ellos crean, comparten y comentan. Y desean hacerlo también en los sitios noticiosos", señala Mark Briggs (2007, p. 40). Bajo este precepto, la detención de Assange fue comentada por ciudadanos que argumentaban a favor y en contra; no solo recibieron la información, sino que también se involucraron.

\section{Ediciones digitales}

El trabajo realizado por los periodistas ya no solo se plasma en una edición impresa. En esta investigación se analizaron las ediciones digitales de los diarios Komsomolskaya Pravda, El País, The New York Times y The Sun. El periodismo está en la web, se ha adaptado a un proceso de cambio en el cual el público es más exigente y quiere la información incluso en tiempo real. Para Pérez (2012), ahora la información no está solo en manos del periodista, sino también del lector, la participación e interacción forma parte de la nueva manera de publicar información. Ya no es solo lo que el medio dice, sino lo que el público tiene para decir al respecto.

\section{Hipervínculos}

La característica principal de todos los diarios analizados es la de los hipervínculos utilizados (Di Palma, 2010, p. 129). El autor los explica como: "Compuesto por una red de nodos o piezas de información creadas y enlazadas con la asistencia de los programas editores, para que luego el lector los pueda recorrer, de acuerdo a sus preferencias".

\section{Metodología y muestra}

Este estudio es de tipo cualitativo como categoría de diseño de investigación que da prioridad a la comprensión y al sentido, en un procedimiento que tiene en cuenta las intenciones, las motivaciones, las expectativas, las razones y las creencias de los individuos (Monje citado en Bravo, 2019, p. 6). También es cuantitativo ya que usa instrumentos de medida, como la estadística, para precisar las observaciones, análisis e interpretación de resultados (Gómez, Deslauriers y Piedrahita citados en Bravo, 2019, p. 6).

Como muestra se tomaron cuatro medios a escala internacional: uno de procedencia rusa (Komsomolskaya Pravda), uno español (El País), uno estadounidense (The New York Times) y uno británico (The Sun), todos analizados desde sus ediciones digitales. El diario Komsomolskaya Pravda fue analizado 7 días en los cuales presentó información sobre Assange, El País (22 días), The New York Times (6 días) y The Sun (14 días). 
Los medios presentaron contenidos a través de 12 notas informativas, 53 crónicas y 17 reportajes, donde profundizaban y explicaban todo lo relacionado a la detención de Julian Assange. Dentro del espacio de opinión se encontraron 1 editorial, 3 columnas y 2 comentarios como posicionamiento de cada medio.

Entre las técnicas empleadas están: a) Revisión documental, donde se recabó información desde el día en que Julian Assange fue detenido (11 de abril), juntando todos los contenidos publicados hasta septiembre. Esta técnica: "Debe considerarse exclusivamente como descripción del contenido y no como descripción formal" (Clauso citado en Bravo, 2019, p. 6). b) Análisis de contenido, conocido como el método para estudiar y analizar las comunicaciones de una forma sistemática, objetiva y cuantitativa (López citado en Bravo, 2019, p. 6). La información encontrada fue analizada desde el enfoque, géneros y términos utilizados.

El proceso se desarrolló en cuatro etapas: primera, recopilación de la información mediante internet; segunda, organización de los contenidos con una matriz de publicaciones generales, en la que se detalla el medio, fecha, titular y enlace, para acceder fácilmente a los contenidos por día de publicación. Tercera, clasificación de la información que, debido a la amplia cantidad de hipervínculos encontrados por nota, se hizo necesaria organizar la información adjunta con una matriz de hipervínculos estableciendo el medio, la nota principal por día y los respectivos subhipervínculos de cada una de ellas, reflejando el titular y detallando si la información es repetida o si está fuera de la fecha de análisis. Cuarta, discusión y presentación de los resultados, complementada con la fundamentación teórica.

Para la recuperación se empleó la matriz de análisis de contenido, descrita como función preparatoria que consiste en entregar a cada investigador la información de primera mano (Galdón citado en Bravo, 2019, p. 7). En ella se aborda lo cualitativo y cuantitativo. La primera parte consta del título, fecha de publicación y recuperación, género periodístico, procedencia de la información, hipervínculos, emplazamiento y elementos de ayuda gráfica y audiovisual. La segunda parte incluye el qué dice y cómo lo dice; esto permitió conocer el enfoque, los géneros y los términos utilizados para el abordaje.

\section{Resultados}

Con el uso de matrices se agrupó la información encontrada, clasificando los medios y obteniendo resultados por cada día en que abordaron temas relacionados con la detención de Julian Assange. De este modo se determinó el enfoque que le dieron, los géneros que usaron dentro de lo informativo y de opinión; también se clasificaron los contenidos que se adjuntaban a las publicaciones en forma de hipervínculos, para saber si se aportaba información nueva o si estaba fuera del tema a investigar. 
Tabla 1. Enfoque de los medios analizados sobre la detención de Assange

\begin{tabular}{l|c}
\multicolumn{1}{c}{ Medio } & $\begin{array}{c}\text { Enfoque } \\
\text { 1. Komsomolskaya contenidos conllevan información y fuentes } \\
\text { Pravda } \\
\text { favorables para Julian Assange. La forma de abordar el } \\
\text { caso se inclina a favor, expuesto desde su labor, aportes } \\
\text { que según el medio ha hecho y su condición como ser } \\
\text { humano. }\end{array}$ \\
\hline 3. The País & $\begin{array}{c}\text { Sus publicaciones manejan un enfoque direccionado } \\
\text { a la imparcialidad, se recogen versiones tanto a favor } \\
\text { como en contra de Assange. El medio tiene perspectivas } \\
\text { variadas, en algunos contenidos lo apoyan, en otros } \\
\text { están en contra y en otros se inclinan a ser imparciales. }\end{array}$ \\
\hline $\begin{array}{c}\text { Se evidencia una inclinación hacia los intereses que } \\
\text { desde el medio existen en las decisiones sobre la } \\
\text { detención del activista, analizando lo que quieren los } \\
\text { países involucrados, bastante cerca de la imparcialidad. }\end{array}$ \\
$\begin{array}{c}\text { Un enfoque negativo para Julian Assange. Los } \\
\text { contenidos tienen información que lo desprestigia, sacan } \\
\text { a la luz datos irrelevantes solo para sumar cosas en su } \\
\text { contra. Dejan claras sus diferencias. }\end{array}$ \\
$\begin{array}{l}\text { Fuentey elaboración propias } \\
\text { The Sun }\end{array}$
\end{tabular}

En la tabla 1 se analiza el enfoque que dieron los diarios desde la forma en que presentaron los contenidos y el tratamiento que tuvo la información.

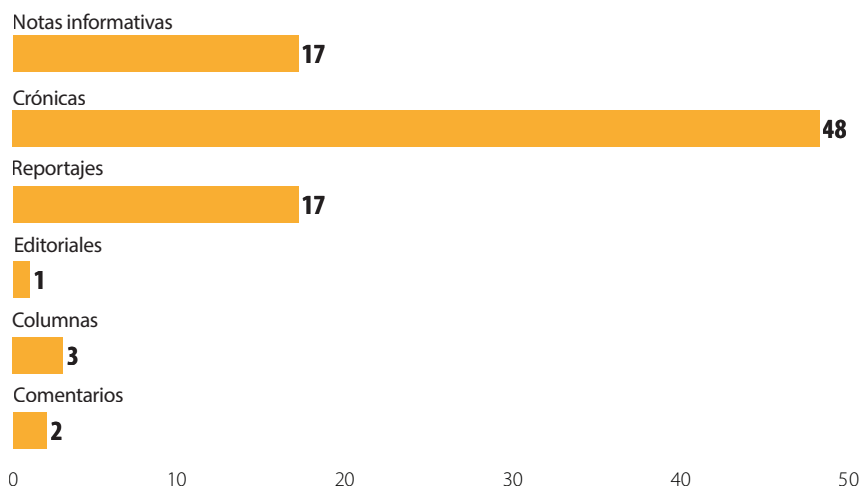

Gráfico 1. Géneros utilizados. Fuente y elaboración propias

El gráfico 1 muestra la suma de los géneros utilizados por los cuatro medios analizados. El género que prevalece es la crónica, seguido de reportajes y notas informativas. Los diarios casi no escribieron columnas, hay pocos comentarios y un solo editorial. 


\begin{tabular}{|c|c|c|c|c|c|}
\hline & Komsomolskaya Pravda & El País & The New York Times & The Sun & \\
\hline Comentarios & 0 & 0 & 1 & 1 & \\
\hline Columnas & 0 & 3 & 0 & 0 & \\
\hline Editoriales & 0 & 1 & 0 & 0 & \\
\hline Reportajes & 0 & 2 & 2 & & 13 \\
\hline Crónicas & 7 & 24 & 3 & & 14 \\
\hline $\begin{array}{r}\text { Notas } \\
\text { informativas }\end{array}$ & 11 & 5 & 0 & 1 & \\
\hline
\end{tabular}

Gráfico 2. Clasificación de géneros por medio. Fuente y elaboración propias

El gráfico 2 representa la cantidad y el tipo de género empleado por cada diario analizado. Komsomolskaya Pravda es el único medio en escribir más notas informativas, los demás coinciden en abordar la información con un mayor número de crónicas.

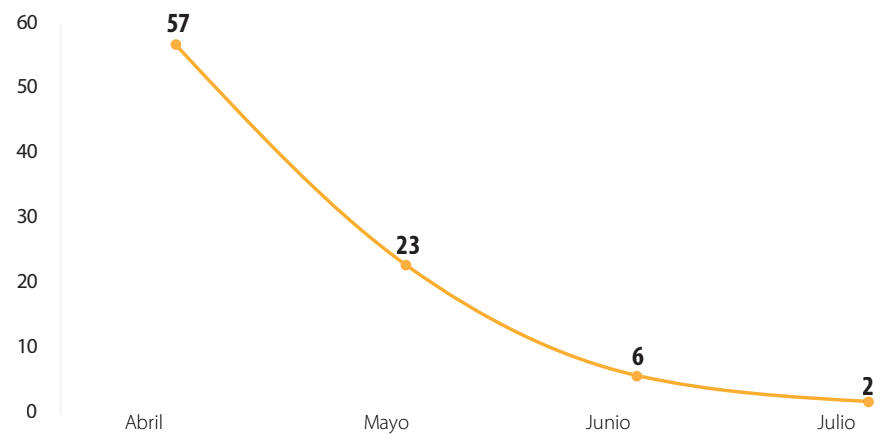

Gráfico 3. Publicaciones de los diarios por mes en 2019. Fuente y elaboración propias

El gráfico 3 ilustra la atención de los medios al abordar el caso de Julian Assange. Numéricamente se evidencia el mes en que Assange protagonizó los diarios y cómo fue decayendo. 


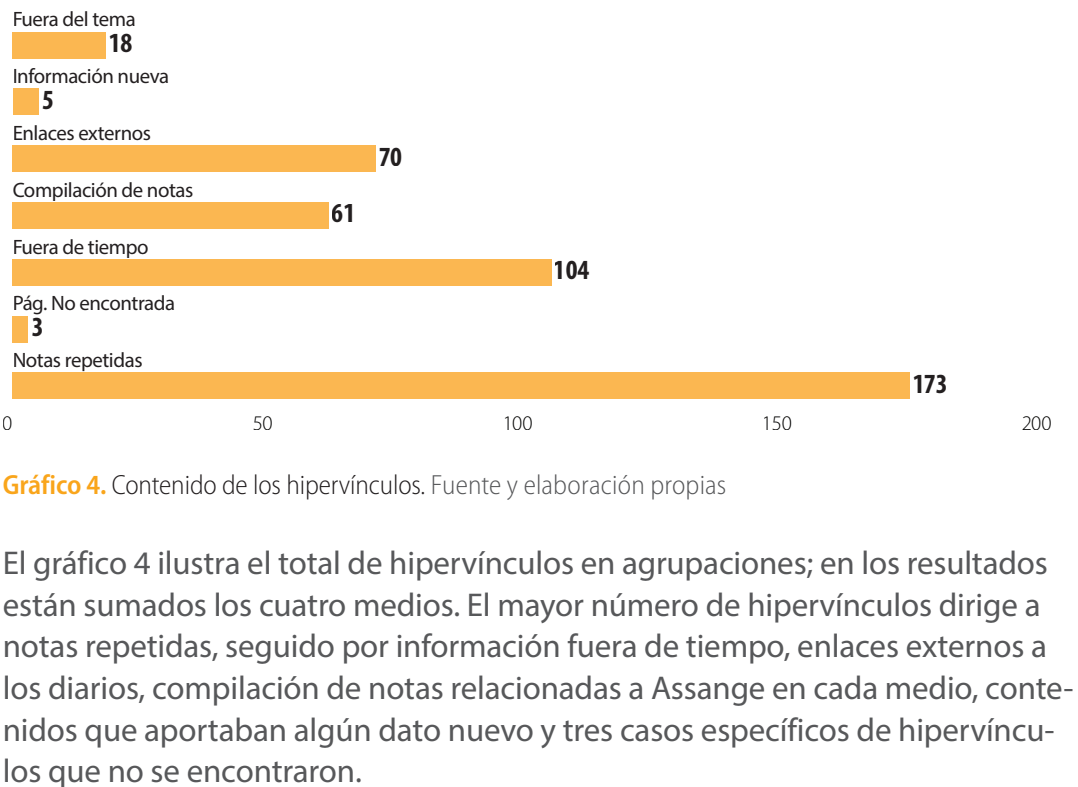

los que no se encontraron.

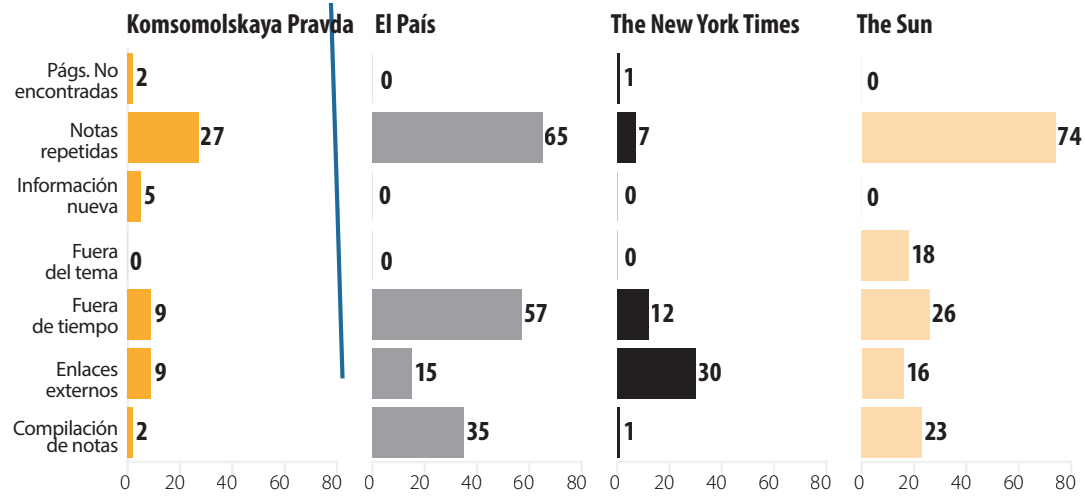

Gráfico 5. Información detallada de los hipervínculos. Fuente y elaboración propias

El gráfico 5 detalla de manera agrupada el contenido que presentó cada diario en sus hipervínculos. Las descripciones son las mismas para todos, solo varía el número de enlaces que empleó cada medio. Únicamente The New York Times tiene más enlaces externos a su edición, los restantes coinciden en mostrar información repetida. 


\section{Discusión}

\section{Enfoque de los medios analizados}

La tabla 1 explica el enfoque que cada medio dio a la información publicada sobre la detención de Julian Assange. Las ediciones digitales que constan en la tabla 1 (numerales 1 al 4) presentan los casos y denuncias que enfrenta en los diferentes países que lo acusan, desde su necesidad de pedir asilo a Ecuador y cómo perdió tal beneficio. Komsomolskaya Pravda dio un tratamiento de la información que señala que solo hacía su trabajo, publicaba contenidos que debían ser analizados y que revelaba situaciones ocultas, dando a entender que toda la situación ligada a su detención se trataba de una venganza por todos quienes se vieron descubiertos por su trabajo en WikiLeaks.

Hay fuentes que hablan a favor del australiano, defendiendo su postura en las investigaciones. Hay versiones positivas como la de Margarita Simonyan (2019), editora del canal ruso $R T$, quien sostiene:

Assange contó cómo funciona el mundo, más precisamente, cómo funciona la hipocresía mundial. Mucho antes de que todos empezáramos a sospechar algo, explicó que Google y otros twitters en este mundo están ahí para a) seguir a todos b) controlar el estado de ánimo de la multitud. (Komsomolskaya Pravda, 11 de abril de 2019)

Asimismo, reunió reacciones sobre la noticia de la detención del australiano. Estas son positivas, una de ellas cita: "La mano de la 'democracia' aprieta la garganta de la libertad" (Maria Zakharova, representante oficial del Ministerio de Asuntos Exteriores de la Federación Rusa - en Facebook) (Komsomolskaya Pravda, 11 de abril de 2019).

En El País se observó que el medio buscó acercarse a la imparcialidad. Relata cómo era la vida de Assange y a qué se dedicaba antes de su asilo político, los problemas legales que ha atravesado y su futuro incierto sobre la extradición. Presenta fuentes a favor y en contra de Assange, se habla desde los logros y aportes, hasta de sus errores e intereses. Se cita al expresidente de la Generalitat de Cataluña, Carles Puigdemont: “Estoy profundamente conmocionado por el arresto de Julian Assange en Londres. Los derechos humanos, y especialmente la libertad de expresión, están siendo atacados una vez más en Europa" (El País, 11 de abril de 2019).

Por otro lado, el criterio completamente opuesto del juez británico, Michael Snow, quien sostuvo: 
Su afirmación de que no ha tenido un juicio justo es una broma, y me temo que es fruto del comportamiento de un narcisista incapaz de pensar más allá de sus propios intereses egoístas. (El País, 12 de abril de 2019).

En The New York Times el enfoque es político, expone la relación que hay entre Ecuador con el hacker desde el cambio de presidente; cómo el exmandatario Rafael Correa acogió al australiano con ciertos beneficios en su gestión. Pero Lenín Moreno dio un giro a la situación, partiendo del cambio de gobierno en la línea política y quitándole a Assange sus ventajas en la embajada, hasta retirarle el asilo. Lo plantea desde varias aristas:

Para sus partidarios, Assange es un mártir y defensor de la libertad de expresión. Para el gobierno de Estados Unidos, es un paria y un lacayo del Kremlim. Pero fue el radical cambio de opinión del gobierno de Ecuador lo que, al final, afectó más su situación. (The New York Times, 11 de abril de 2019)

También pone a consideración del lector si es una coincidencia o un movimiento político, que Julian Assange haya sido expulsado de la embajada luego de 17 días en que él haya publicado fotos personales de las vacaciones del expresidente de Ecuador, Lenín Moreno.

The Sun aborda no solo el arresto sino sus problemas con la justicia, profundizándolas como algo importante. Las fuentes que utilizan son notoriamente negativas, acompañadas con las opiniones de quienes agradecen que Ecuador "entregara" al australiano a la justicia. Cita al primer ministro que dijo a los Comunes: "Estoy seguro de que toda la casa recibirá con agrado la noticia de que la Policía Metropolitana ha arrestado a Julian Assange" (The Sun, 11 de abril de 2019).

Se encontraron fuentes a favor, pero eran empañadas con algún tipo de información extra que desvía la atención de sus aportes favorables.

Su enfoque está también en las notas que publicaron sobre la vida personal de Assange, resaltando incluso en titulares aspectos irrelevantes que no estaban relacionados a su detención: "Soy un cerdo de cabeza intelectual", haciendo referencia a un sitio de citas donde Assange se describía de esa manera.

\section{Géneros periodísticos empleados}

Entre los cuatro diarios analizados el género que prevalece es la crónica (53 en total), en segundo lugar el reportaje (17), en tercer lugar la nota 
informativa (12), en cuarto lugar la columna (3), en quinto lugar el comentario (2), y en sexto lugar el editorial (1). El gráfico 1 explica los géneros utilizados; el gráfico 2, la clasificación de géneros por medio.

Komsomolskaya Pravda presenta más notas informativas, seguidas de crónicas con enfoque informativo e interpretativo. En ellas hay detalles del ambiente en el que vivió Assange durante sus casi siete años (2487 días) en la embajada de Ecuador en Londres, las medidas de la habitación en la que residía, la relación con el personal de la policía británica, las privaciones luego de la llegada del expresidente ecuatoriano Lenín Moreno, restringiendo las visitas, el impedimento del uso de internet y la vigilancia que recibía tanto por el personal como por las cámaras de seguridad. Planteaban un escenario desfavorable para Assange, como un prisionero que fue perdiendo sus derechos.

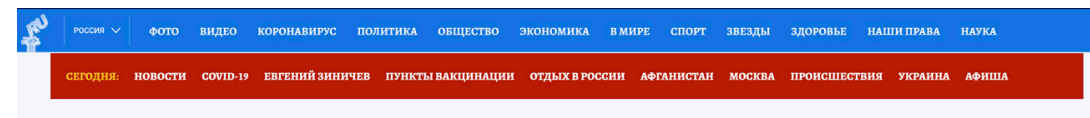

B

\section{Седовласый старик: арестованный Ассанж шокировал своим видом}

Основателя WikiLeaks взяли под стражу в Лондоне

Ольга ЛИБГАРдТ ПОдПисаться

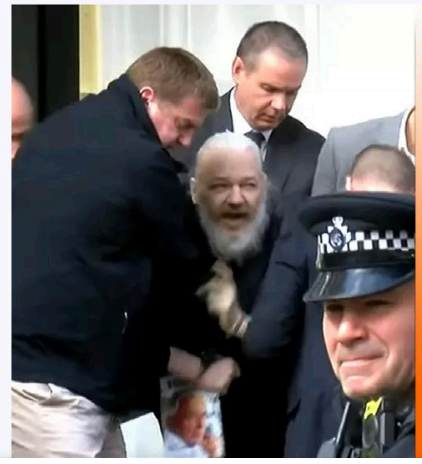

„поделиться

\section{Э КОММЕНТАРИИ (84)}

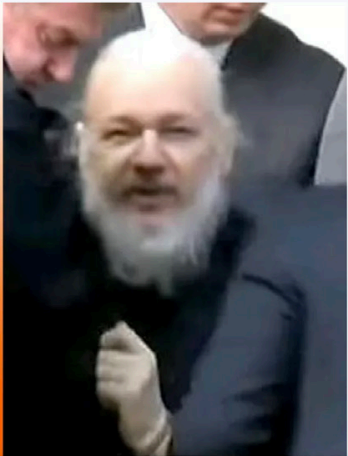

Imagen 2. Ejemplo del contenido de Komsomolskaya Pravda publicado del 11 de abril de 2019. Captura de pantalla.

El País desarrolla un mayor número de crónicas, seguido de notas informativas y reportajes, los contenidos son mayormente informativos con versiones variadas, tanto positivas como negativas, citan por ejemplo opiniones de 
personajes como el expresidente de la Generalitat de Cataluña. De igual manera, exponen las declaraciones de Michael Snow, el juez británico ante quien Assange compareció en 2019; el medio buscó dar un equilibrio y aclarar si la situación era meramente delictiva o si existían conflictos políticos de por medio.

En los contenidos de género de opinión, por un lado, se lo expone como un héroe por los descubrimientos y aportes que según el periodista ha hecho por la sociedad. Por el otro, se explica su situación desde la parte legal y las decisiones que se podrían tomar frente al hecho, pero en sí se identifica un llamado a no centrarse en el fundador de WikiLeaks para apoyarlo o no, sino en las irregularidades que publicó para que estas sean tratadas por la ley y las personas que hayan sido descubiertas en cosas anormales, paguen.

$\begin{array}{ll}\text { EL PAÎS } & \text { OPINIÓN } \\ \text { EDITORIAL } & \text { i }\end{array}$

\section{Assange, detenido}

El cofundador de WikiLeaks tiene derecho a un proceso justo y transparente
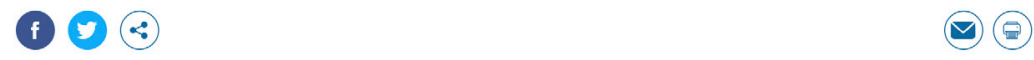

EL PAIS $y$

11 ABR 2019 - 17:00 ECT

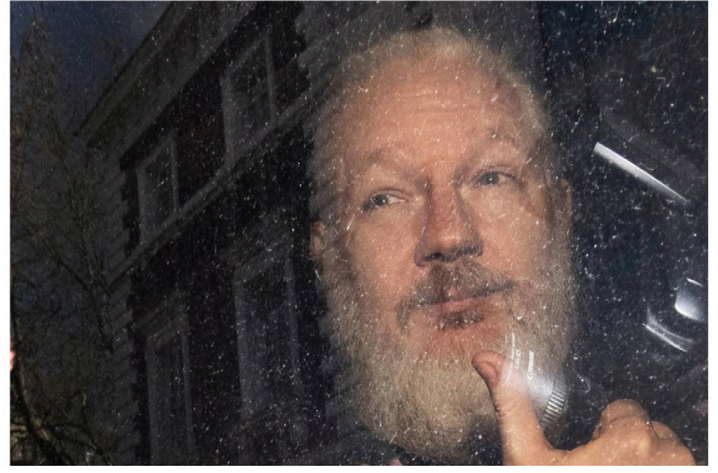

Assange saluda desde el interior del coche de policfa que lo traslada, este jueves en Londres. VICTORIA
JONES / AP

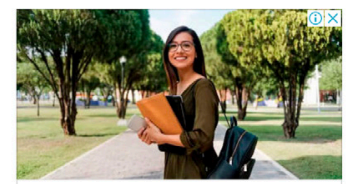

\section{Maestría con titulo europeo}

Maestrías con

modalidad totalmente

Online. Reconocidas por SENESCYT en Ecuador.

Infórmate

UHemisferios IMF

Imagen 3. Ejemplo de una publicación de opinión del diario El País de España del 11 de abril de 2019. Captura de pantalla.

En las crónicas y reportajes, The New York Times evidencia un enfoque alrededor de los intereses de quienes se vinculan en el caso. Es el diario que 
más se acerca a la imparcialidad; aborda información en todas las direcciones buscando un equilibrio. Interesa al lector en el cambio de relación entre Ecuador y Assange: qué quieren cada uno; por qué Estados Unidos y Suecia quieren su extradición; por qué unos se pronuncian al respecto y otros no. Abre el abanico de posibilidades, las palabras que usa las expone según la consideración que le tienen a Assange.

\section{Ehe Aiew dork Eimes}

INTERNACIONAL

\section{Arrestan a Julian Assange en la embajada de Ecuador en Londres}

f) $(6)$

Por Eilleen Sullivan y Richard Pérez-Peña

11 de abril de 2019

Read in English
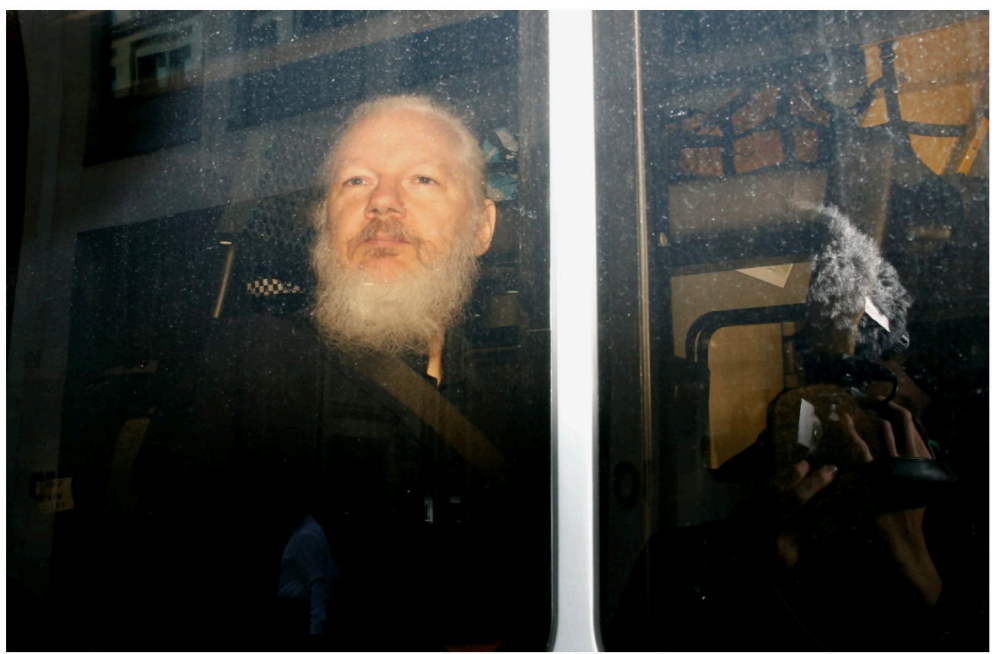

Julian Assange, el fundador de WikiLeaks, en una camioneta de la policía afuera de la embajada de Ecuador en Londres después de su arresto este 11 de abril Henry

Imagen 4. Ejemplo del contenido de The New York Times publicado el 11 de abril de 2019. Captura de pantalla. 
En el caso de The Sun, sus contenidos se encuentran mayormente en crónicas y reportajes. Este diario aprovecha la extensión de esos géneros para hacer un recuento de la situación de Assange y añadir versiones, notablemente en contra. Cuando incluyen fuentes favorables las citan textualmente y atribuyen, aunque suelen ir seguidas de algún tipo de información que desvíe la atención del aporte central. Por ejemplo, Pamela Anderson es la famosa que más se pronuncia al respecto, defiende a Assange abiertamente; el medio expone su opinión, pero al mismo tiempo cuenta que había rumores que decían que ella era su amante; así se le da un giro a la información.

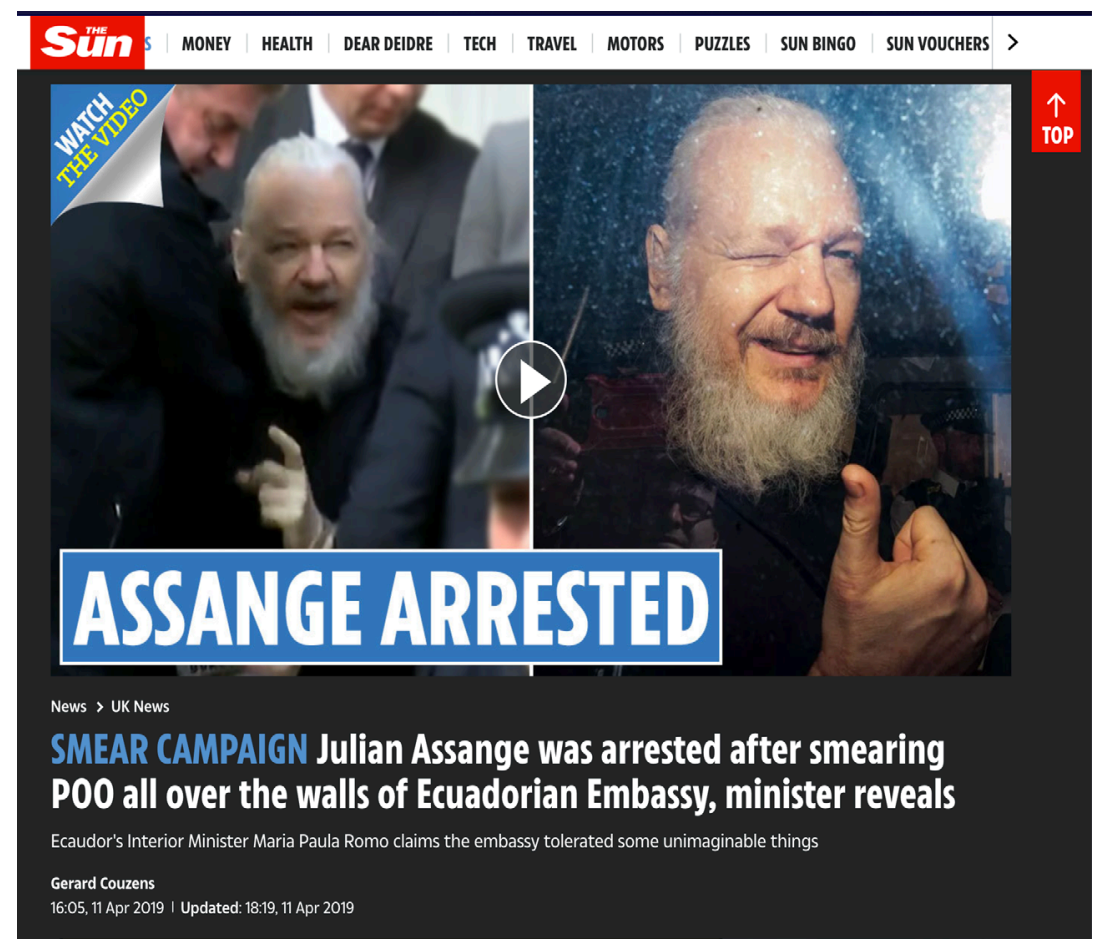

Imagen 5. Ejemplo de las publicaciones de The Sun del 11 de abril de 2019. Captura de pantalla.

\section{Publicaciones de los diarios por mes}

Los cuatro medios (ver Gráfico 3) reflejan mayor número de publicaciones en abril, 57 en total. En ese mes detuvieron a Assange, los diarios recogían información de las personas y países que opinaban al respecto, consultaban a expertos en el tema y analizaban el futuro incierto de este personaje. En abril se aprovechó el tema para explicar su situación legal con Estados Unidos y Suecia, los problemas que había tenido y sus argumentos de 
defensa. La terminación del asilo político también hizo que los medios analizaran la relación entre Assange y Ecuador; se expusieron las supuestas razones que Lenín Moreno, presidente ecuatoriano, había tenido para dar fin a los beneficios del huésped.

En mayo, las noticias sobre el australiano se redujeron a 23; en junio su situación casi no apareció en los medios, seis apenas; y para julio su caso fue prácticamente descartado de la agenda, encontrando solo dos.

Komsomolskaya Pravda inició sus publicaciones aquel 11 de abril con una crónica titulada: "Lenin se rindió a Assange: el fundador de WikiLeaks fue arrestado en la embajada de Ecuador en Londres". Allí se habla de la decisión de Ecuador de retirar el asilo político a Assange, las razones que dio el presidente ecuatoriano y las reacciones que trajo consigo, citando opiniones a favor de Assange y juzgando el fin de su estadía en la embajada.

El diario que más se mostró en contra, The Sun, tiene como nota final (del tiempo de análisis) la crónica del 11 de julio: “Cloo Sabía, Amal Clooney 'le dijo a Julian Assange cómo evitar el arresto y huir de Gran Bretaña', muestran documentos filtrados". Según la información de Amal Clooney, quien en su momento como abogada de Assange le sugirió a él ser ministro de Tecnología de Ecuador, considerando que esto sí era posible. Esta situación fue descubierta por documentos filtrados, catalogándola como una manera de querer sabotear la decisión de Reino Unido de encarcelarlo.

\section{Clasificación de hipervínculos}

La interpretación ilustrativa se encuentra plasmada en el gráfico 4 (Total de la información de hipervínculos) y el gráfico 5 (Información de los hipervínculos por medio). Komsomolskaya Pravda tuvo un total de 18 publicaciones con 54 hipervínculos, El País, 35 publicaciones y 172 hipervínculos; The New York Times, 6 publicaciones y 51 hipervínculos; finalmente, The Sun, 29 publicaciones y 157 hipervínculos.

El País es el medio que tuvo más contenidos, y desde el seguimiento y la clasificación de cada uno de ellos, la mayoría de información adjunta en las notas principales son repetidas, tomadas del mismo diario, pero publicadas en días diferentes; esto para que el lector pueda empaparse más del caso y acceder fácilmente a situaciones explicadas con anterioridad. El contenido de hipervínculos que le sigue es de notas fuera del tiempo de investigación, publicadas hace años o a inicios de 2019. En El País también hay hipervínculos que direccionan al lector a un cúmulo de notas del propio medio sobre el caso de Assange, y notas sobre los dos últimos presidentes de Ecuador. El número de hipervínculos en la categoría enlaces 
externos al medio hace referencia a toda la información que es tomada de otras personas, medios o de sitios web, así como de aspectos que constan en redes sociales de perfiles de Twitter e Instagram, WikiLeaks y personas relacionadas. Dichos enlaces muestran opiniones y posturas sobre el trabajo que Assange publicaba, haciendo énfasis en que el poder lo quería silenciar ya que él mostraba sus irregularidades; parte de esos enlaces también conectaban con notas sobre las denuncias y acusaciones que Assange tenía pendiente en otros países, y pedidos a la justicia para que él enfrente los cargos.

The Sun se ubica segundo en la cobertura de la detención, tomando en consideración que procede del país en que se desarrollaron los hechos. La mayoría de sus hipervínculos repiten la información sacada por ellos mismos; como en el medio anterior también hay contenidos fuera del tiempo de investigación, compilación de notas del diario, enlaces externos, pero en este se añade la aparición de notas fuera del tema, entre ellas la del compañero de celda de Assange que explica su peligrosidad.

The New York Times y Komsomolskaya Pravda tienen las mismas características explicadas, a excepción del segundo, que en cinco notas la información es nueva y aporta a la lectura.

\section{Conclusiones}

Con base en la discusión de resultados de las 88 publicaciones escogidas como muestra de los cuatro medios analizados (Komsomolskaya Pravda, El País, The New York Times y The Sun) sobre la detención de Julian Assange, se llegó a varias conclusiones.

Los resultados respondieron a la pregunta de investigación: ¿Cómo se realiza el tratamiento de la información sobre la detención de Julian Assange en las ediciones digitales Komsomolskaya Pravda, El País, The New York Times y The Sun? El contenido de la tabla 2 expone el enfoque que tuvieron los medios analizados: Komsomolskaya Pravda abordó el hecho con versiones y fuentes que defienden a Assange; también presenta información negativa, pero le da mayor cabida a quienes aportan con algo a su favor. El País publicó notas positivas para Assange con fuentes y argumentos, además expuso contenidos negativos sobre situaciones y criterios que no le convenían; en opinión lo apoyó en cosas que consideró hizo bien, como a la vez lo criticó por errores en su actuar; el medio buscó acercarse a la imparcialidad. The New York Times fue el medio que más trató de equilibrar el tratamiento de la información: presentó a Assange como era considerado desde sus seguidores y detractores, su enfoque giró en torno a la relación que él mantenía con Ecuador, intereses 
políticos de Estados Unidos, WikiLeaks, Julian Assange, el Gobierno ecuatoriano y todos quienes se involucraron en el hecho; fue el que más cerca estuvo de la imparcialidad. The Sun se mostró abiertamente en contra de Assange, sus publicaciones eran negativas, daba espacio a quienes hubiesen opinado algo malo sobre Assange, incluso indagó en su vida personal mostrando información irrelevante que no aportaba al tema. Queda demostrado que los medios analizados no fueron cien por ciento imparciales al abordar el hecho, su inclinación pudo notarse en el tratamiento de la información.

El objetivo planteado se cumplió. Los géneros periodísticos empleados por los medios al abordar la detención de Assange fueron identificados; la crónica prevaleció siendo el género más utilizado, con un carácter informativo e interpretativo; este género primó debido a que los medios emitían juicios de valor a la hora de hablar sobre el trabajo que Assange había realizado y publicado en WikiLeaks. Luego estuvo el reportaje que, por su extensión y al ser digitales, adjuntaba evidencias que complementaban y enriquecían la información como documentos y enlaces. La nota informativa es el tercer género más empleado; allí explicaban la detención de Assange acompañada con fuentes. En menor cantidad hubo también géneros de opinión, un editorial, columnas y comentarios. Al hablar de información se pensaría que las notas informativas serían las protagonistas, pero tras el análisis de contenido se encontró que no es así: las notas que buscaban informar eran menores considerando que la situación de Assange despertó el interés del mundo y que los medios tomaron una posición al respecto, hecho que la crónica les permitió indagar y profundizar.

La detención de Assange fue cubierta con mayor notoriedad durante abril, mes en el que sucedieron los hechos, con 57 publicaciones en total, en mayo se redujo a 23, junio solo seis y en julio casi se dio por terminado el tema, con dos contenidos. El diario que se apresuró a cubrir el caso fue Komsomolskaya Pravda, explicando la decisión del expresidente de Ecuador de dar por terminado el asilo político de Assange, y las consecuencias y reacciones que eso provocó. The Sun, por su parte, es el medio que subió información hasta julio, mes en que la noticia casi no presentaba coberturas; su nota final dentro del tiempo de análisis habla del plan que tuvo en su momento la exabogada del australiano para que se librara de la cárcel de Reino Unido. Esto refuerza la inclinación de ambos medios explicada con anterioridad: Komsomolskaya Pravda a favor y The Sun, en contra. 
Los cuatros diarios tuvieron como principal característica el uso de hipervínculos, sumando 434 en total, superando las 88 publicaciones hechas por los medios. Los hipervínculos direccionaban al lector a información referente al tema tal como se explica en la tabla 4, gráficos 4 y 5. 


\section{Referencias}

Arresto de Julian Assange: ¿por qué detienen ahora al fundador de WikiLeaks tras 7 años refugiado en la embajada de Ecuador en Londres? (2019, 11 de abril). BBC News Mundo. https://www.bbc.com/mundo/ noticias-internacional-47897043

Assange contó cómo funciona la hipocresía mundial, mucho antes de que todos empezáramos a sospechar algo. (2019, 11 de abril). Komsomolskaya Pravda. https://www.kp.ru/daily/26965/4020259/

Astudillo, F. y Buitrón, R. (2005). Periodismo por dentro: Una pausa en medio del vértigo. Editorial Quipus / CIESPAL Ecuador.

Bravo, J. (2019). Leonidas Proaño: Ejercicio periodístico para el cambio social. Risti.

Briggs, M. (2007). Periodismo 2.0: Una guía de alfabetización digital. Centro Knight para el Periodismo en las Américas / Universidad de Texas.

Cambridge, E. (2019, 11 de abril). Que ganador: Julian Assange se enfrenta a un año en la cárcel británica después de un dramático arresto en la embajada ecuatoriana, ya que Estados Unidos exige que lo entreguemos. The Sun. https://www.thesun.co.uk/news/8839586/ julian-assange-wink-thumbs-up-arrest-embassy/

Di Palma, G. (2010). Introducción al periodismo: Internet y tecnología digital, prensa gráfica, radio y TV. Editorial Brujas.

Edo, C. (2009). Periodismo informativo e interpretativo: El impacto de internet en la noticia, las fuentes y los géneros. Alfaomega Grupo Editor, S.A. de C.V.

Erlenger, S. y Casey, N. (2019, 11 de abril). Siete años en una embajada: el extraño aislamiento de Julian Assange. The New York Times. https://www. nytimes.com/es/2019/04/11/assange-embajada-ecuador/?rref=collection $\% 2$ Fsectioncollection\%2Fnyt-es

González, S. (2009). Periodismo de opinión y discurso. Editorial Trillas, S.A de C. V.

Julian Assange afronta un año de cárcel antes de su extradición a Estados Unidos. (2019, 12 de abril). El País. https://elpais.com/internacional/2019/04/11/actualidad/1554975440_843068.html?fbclid=IwAR1 fx5ClFDfn5iD5HDLcHJhoNLBYdzyHxl1EkTG84Ku4mEBAHHgPaUJLGug 
Lenin se rindió a Assange: el fundador de WikiLeaks fue arrestado en la Embajada de Ecuador en Londres. (2019, 11 de abril). Komsomolskaya Pravda. https://www.kp.ru/daily/26965/4020141/

Pérez, L. P. (2012). Las nuevas reglas de la participación del público en la prensa digital: Análisis de siete diarios europeos de referencia/The new rules of audience participation in digital media. analysis of seven european newspapers. Estudios sobre el mensaje periodístico, 18(1), 193-210. https://search.proquest.com/docview/1033194509?accountid=36757

Ramírez, J. (2018). Método histórico: Características, etapas, ejemplos. https:// www.lifeder.com/metodo-historico/

RT. (2019, 12 de abril). ¿Cómo vivía Assange en la Embajada de Ecuador? [Archivo de video]. RT. https://actualidad.rt.com/ video/311566-como-vivia-assange-embajada-ecuador

Simonyan, M. (2019, 11 de abril). Assange contó cómo funciona la hipocresía mundial, mucho antes de que todos empezáramos a sospechar algo. Komsomolskaya Pravda. https://www.kp.ru/daily/26965/4020259/

Talavera, M. d. M. L. (2012). La ética periodística en el tratamiento informativo de la inmigración. Cuadernos de Información y Comunicación, 17, 339-354. https://www.proquest.com/ docview/1425872204?accountid=36757\&forcedol=true

Torra y Puigdemont critican la detención de Assange y agradecen su apoyo al 1-0. (2019, 11 de abril). El País. https://elpais.com/politica/2019/04/11/ actualidad/1554986750_067667.html 